\title{
Futbol: lenguaje formal y simulación computable
}

\author{
Jonathan Téllez Girón, Matías Alvarado \\ Centro de Investigación y de Estudios Avanzados del Instituto Politécnico Nacional, \\ CDMX, México \\ jtellez@computacion.cs.cinvestav.mx, matias@cs.cinvestav.mx
}

\begin{abstract}
Resumen. En este artículo el lenguaje formal del futbol soccer es generado por una gramática libre de contexto, traducción de las reglas del juego, tal que las cadenas del lenguaje describen cualquier combinación posible de jugadas correctas, y así, un partido completo. La interacción entre los jugadores en el campo de juego conlleva un sistema de cómputo concurrente. Mediante centenares de simulaciones computacionales se valora el funcionamiento y desempeño del modelo y el sistema planteado. En particular, se aplica a las formaciones clásicas de un equipo al jugar bajo cierto esquema táctico y estratégico. Se analiza la aplicación del equilibrio de Nash para la selección de estrategias en el juego.
\end{abstract}

Palabras clave: Futbol, estrategias, equilibrio de Nash, sistema concurrente.

\section{Football: Formal Language and Computable Simulation}

\begin{abstract}
In this article, the formal language of soccer is generated by a contextfree grammar, translation of the rules of the game, such that the language chains describe any possible combination of correct plays, henceforth a complete match. The interaction among the players on the playfield it comprises a concurrent computation system. Through hundreds of computational simulations the model pertinence and the system performance are evaluated. Particularly, it applies to the classic formations of a team playing under a certain tactical and strategic scheme. The applicability of Nash equilibrium to selection of strategies in the game is outlined.
\end{abstract}

Keywords: Football, strategies, Nash equilibrium, concurrent system.

\section{Introducción}

El futbol (del inglés football) es un juego estratégico de equipo, muy popular a nivel mundial. Se juega en una cancha de césped de 120 x $60 \mathrm{~m}$, con arcos o porterías en cada lado del campo, entre dos equipos de 11 jugadores cada uno; un partido inicia con 
el balón a media cancha y un jugador del equipo que ganó el bolado para dar el primer toque al balón lo pasa a un compañero. Los roles de juego, acorde a su posición en la cancha respecto a la portería propia son delanteros, medios, defensas, y el portero, siendo la misión del portero evitar anotaciones de gol en su portería, y sólo él tiene permitido el manejo del balón con manos y brazos. Sobre el césped los jugadores deben conducir el balón con los pies y pueden controlarlo, asimismo, con piernas, cuerpo y cabeza, pero no con brazos ni manos, salvo para los saques de banda y el portero respetando las reglas asignadas. A base de conducción individual y pases del balón entre compañeros y de esquivar a los rivales, deben buscar introducir el balón en la portería rival para anotar goles, y gana el partido el equipo que anota mayor número de goles.

Las estadísticas de este deporte junto con las técnicas de predicción de resultados son de gran interés para entrenadores, jugadores, empresarios y aficionados. En este artículo se presenta la gramática que genera el lenguaje formal de este juego, similarmente a como se hace en el béisbol (BB) [1] y el futbol americano (FA) [2]. Este modelo da pie a la simulación algorítmica del juego y al sistema concurrente para ejecutar la continuidad interactiva entre los jugadores: cada jugador es un proceso hilo en el sistema computacional concurrente (SCC).

Se desarrollan las reglas gramaticales para cada jugador, que son leídas por el autómata finito no determinista correspondiente. La transición entre jugadas, así como la lógica de acuerdo a las condiciones del campo, se definen mediante las reglas de la gramática formal, que traducen las reglas del futbol. La interacción de jugadores es a través de la sección crítica del sistema, es decir, las variables y recursos en común entre los hilos. Las instancias particulares del sistema concurrente utiliza la ocurrencia promedio de jugadas, dadas a su vez en función del rol en la cancha: portero, defensa, medio o delantero. Las formaciones más usuales para un equipo son: 4-4-2, 5-3-2, 4-33 , correspondientes al número de defensas, mediocampistas y delanteros. En algunos ejemplos se utilizan datos estadísticos de la Liga Española en el torneo 2015-2016, en particular de jugadores destacados en equipos con mejor posición en la tabla de la liga. El futbol presenta una dinámica presenta una dinámica diferente respecto al BB y al FA, dada una mayor continuidad de interacción no pausada. Por conveniencia, se limitan cada intervalo de tiempo en el sistema a un minuto de juego por jugada, $\mathrm{t}=$ $1, \ldots, \mathrm{T}$, donde $\mathrm{T}=90$ es el límite regular de un partido oficial de minutos de juego. En cada intervalo cada jugador decide que jugada realiza.

En la Sección 2 se desarrolla la gramática y el lenguaje formal del futbol, en la Sección 3 el sistema de cómputo concurrente y en la Sección 4 se da un análisis inicial de cómo aplicar el equilibrio de Nash para la selección de estrategias. La sección 5 da cabida a la Discusión y se cierra el artículo con la Sección de Conclusiones.

\section{Lenguaje Formal}

El alfabeto $\sum$ es un conjunto finito, no vacío de letras o símbolos. Formalmente un autómata finito es la 5-tupla:

$$
\left\langle Q, \Sigma, \delta, q_{0}, F\right\rangle
$$


donde:

- $Q$ : Conjunto finito no vacío de estados.

- $\Sigma$ : El alfabeto

- $\delta: \mathrm{Q} \times \Sigma \rightarrow \mathrm{Q}$ La función de transición que especifica a que estado pasa el autómata desde el estado actual al recibir un símbolo de entrada.

- $q_{0} \in Q$ es el estado inicial del autómata

- $F \subset Q$ es el conjunto de estados finitos del autómata

La gramática formal es la 4-tupla:

$$
G=\left\langle\Sigma_{T}, \Sigma_{N}, S, P\right\rangle,
$$

donde:

- $\Sigma_{T}$ es el alfabeto de símbolos terminales

- $\Sigma_{N}$ es el alfabeto de símbolos no terminales

- $S \in \Sigma_{N}$ es el axioma, símbolo inicial con el que la inicia la regla inicial.

- $P$ es el conjunto de reglas de producción de la forma $u::=v$, donde $u=$ $x A y, x, y, v \in\left(\Sigma_{T} \cup \Sigma_{N}\right)^{*}$ y $A \in \Sigma_{N}$.

En la gramática una producción es de la forma $A::=v$, donde $v=\left(\Sigma_{T} \cup \Sigma_{N}\right)^{+}$. Puede contener la regla $S:=\alpha$, donde $S$ es el axioma, si la gramática no es recursiva en $S$. A los lenguajes generados por este tipo de gramática se les denomina lenguajes independientes (libres) del contexto (l.i.c.).

En la Tabla 1 se muestran las reglas de la gramática así como de cadenas de acciones o estrategias básicas del lenguaje del futbol, generadas a partir de la gramática independiente del contexto.

Tabla 1. Reglas de producción de gramática formal para un jugador en el futbol.

\begin{tabular}{cl}
\hline \multicolumn{1}{c}{ Regla } & \\
$P \rightarrow$ go $G$ & Jugador anota gol \\
$P \rightarrow$ rea $P$ & Jugador acierta un regate \\
$P \rightarrow \operatorname{rep} P$ & Jugador recibe penalti con balón \\
$P \rightarrow$ far $P$ & Jugador recibe falta con balón \\
$P \rightarrow$ fap $P$ & Jugador realiza falta con el balón \\
$P \rightarrow$ fuj $F J$ & Jugador saca el balón del campo de juego \\
$P \rightarrow d S P$ & Jugador despeja el balón \\
$P \rightarrow$ pl $S P$ & Jugador realiza un pase largo \\
$P \rightarrow$ pc $S P$ & Jugador realiza un pase corto \\
$P \rightarrow$ ce $S P$ & Jugador realiza un centro \\
$P \rightarrow$ t $S P$ & Jugador realiza un tiro \\
$P \rightarrow$ as $S P$ & Jugador asiste a otro jugador \\
$P \rightarrow$ ref $S P$ & Jugador falla un regate \\
$S P \rightarrow$ far $S P$ & Jugador recibe falta sin balón \\
$S P \rightarrow$ blse $S P$ & Jugador bloquea el balón
\end{tabular}




\begin{tabular}{cl}
\hline \multicolumn{1}{c}{ Regla } & \multicolumn{1}{c}{ Descripción } \\
\hline$S P \rightarrow$ db $S P$ & Jugador bloquea un disparo \\
$S P \rightarrow$ pec $S P$ & Jugador comete un penalti \\
$S P \rightarrow \operatorname{rep} S P$ & Jugador recibe penalti sin balón \\
$S P \rightarrow \operatorname{mac} F$ & Jugador comete mano \\
$S P \rightarrow r P$ & Jugador recupera el balón \\
$S P \rightarrow i P$ & Jugador intercepta el balón \\
$S P \rightarrow$ blce $P$ & Jugador bloquea el balón \\
$F J \rightarrow$ eer $S P$ & Jugador pierde el balón al sacarlo del campo \\
$F \rightarrow$ adv $S P$ & Jugador recibe advertencia \\
$F \rightarrow \operatorname{sdu} T A$ & Jugador recibe tarjeta amarilla \\
$F \rightarrow \operatorname{sdd} S T A$ & Jugador recibe doble tarjeta amarilla \\
$F \rightarrow \operatorname{sf} T R$ & Jugador recibe tarjeta roja \\
$T A \rightarrow$ er $S P$ & Jugador con tarjeta amarilla pierde el balón \\
$S T A \rightarrow \operatorname{sa} T R$ & Jugador acumula tarjetas recibiendo tarjeta roja \\
$T R \rightarrow \exp X$ & Jugador es expulsado del juego \\
\hline
\end{tabular}

Con base en la gramática formal definida y las reglas de producción es posible generar cadenas de juego. Sin embargo como se mencionó previamente, las cadenas de juego generadas por la gramática describen el juego de un solo jugador. El sistema concurrente se encarga de simular la interacción entre las cadenas de jugadas, a través de la sección crítica del sistema. La concurrencia se administra iterativamente por todos los hilos de jugador, verificando en cada tiempo de juego las jugadas realizadas por el resto de los jugadores, de tal manera que se determinan las jugadas posibles. La comprobación iterativa mencionada mantiene el realismo de las cadenas para todos los jugadores. Un ejemplo de las cadenas se puede ver en la Tabla 2. En la Tabla 3 se ejemplifica una cadena de juego para una anotación.

Tabla 2. Cadenas de jugadas generadas en el SCC utilizando estadísticas/probabilidades de jugadores reales.

\begin{tabular}{ccccccccc}
\hline \multirow{2}{*}{ Jugador } & \multicolumn{10}{c}{ Tiempo(t) } \\
\cline { 2 - 9 } & $\mathbf{1}$ & $\mathbf{2}$ & $\mathbf{3}$ & $\mathbf{4}$ & $\mathbf{5}$ & $\mathbf{6}$ & $\ldots$ & $\mathbf{9 0}$ \\
\hline Andrés Iniesta & $f a p$ & $a d v$ & $m v s b$ & $m v s b$ & $m v s b$ & $b l c e$ & $\ldots$ & $f a p$ \\
Lionel Messi & $p c$ & $m v s b$ & $f a p$ & $a d v$ & $m v s b$ & $m v s b$ & $\ldots$ & $p c$ \\
Luka Modric & $m v s b$ & $m v s b$ & $m v s b$ & $r$ & $p c$ & $m v s b$ & $\ldots$ & $m v s b$ \\
Cristiano Ronaldo & $m v s b$ & $m v s b$ & $m v s b$ & $m v s b$ & $m v s b$ & $m v s b$ & $\ldots$ & $m v s b$ \\
\hline
\end{tabular}

Consideraciones y restricciones: cada equipo intenta anotar goles y evitar que el equipo rival los anote, y gana el que más anote. Un equipo de futbol define formación ofensiva y defensiva, y un estilo de juego, definido por el entrenador. Para triunfar, el diseño y uso de la combinación de estrategias individuales es cada vez más usual en el futbol competitivo. Una estrategia colectiva adecuada incrementa la probabilidad del triunfo. Con base en la estrategia elegida se define el curso de acciones del equipo en un partido. El modelado formal de este deporte multijugador da sustento al razonamiento estratégico del juego y la selección de estrategias. En [3] cada intervalo de tiempo $(t, t+1)$ es un minuto del partido, $t=0, \ldots, T=90$; en cada $t$ solo puede 
Futbol: lenguaje formal y simulación computable

anotarse un gol o realizar una expulsión, y no se consideran factores externos que afectan al resultado del partido. Estas restricciones las incluimos en nuestra propuesta.

Tabla 3. Cadenas de jugadas representando una anotación en el SCC utilizando estadísticas/probabilidades de jugadores reales.

\begin{tabular}{cccccccc}
\hline & \multicolumn{7}{c}{ Tiempo(t) } \\
\cline { 2 - 8 } & $\ldots$ & $\mathbf{1 4}$ & $\mathbf{1 5}$ & $\mathbf{1 6}$ & $\mathbf{1 7}$ & $\mathbf{1 8}$ & $\ldots$ \\
\hline Andrés Iniesta & $\ldots$ & $m v s b$ & $m v s b$ & blse & $m v s b$ & fap & $\ldots$ \\
Lionel Messi & $\ldots$ & $b l c e$ & $t$ & $m v s b$ & $m v s b$ & $m v s b$ & $\ldots$ \\
Luka Modric & $\ldots$ & $m v s b$ & $f a p$ & $a d v$ & $m v s b$ & $m v s b$ & $\ldots$ \\
Cristiano Ronaldo & $\ldots$ & $m v s b$ & $d b$ & rea & $t$ & $g o$ & $\ldots$ \\
\hline
\end{tabular}

\section{Concurrencia}

Un proceso computacional, proceso a partir de ahora, es una actividad asíncrona susceptible de ser asignada a un procesador, y es el elemento básico para diseñar y ejecutar un programa. Los estados de un proceso son: (1) ejecución: utilizando el CPU; (2) listo: temporalmente detenido para permitir la ejecución de otro proceso; (3) bloqueado: imposibilitando de ejecutarse hasta que un evento externo lo habilite.

Dos procesos son concurrentes cuando existe un solapamiento en la ejecución de sus instrucciones. Un programa concurrente es un conjunto de procesos ejecutables de manera simultánea. A la porción de código que queremos se ejecute de forma indivisible se le llama sección crítica. Las secciones críticas del sistema se ejecutan en exclusión mutua, y solo uno de los procesos debe estar en la sección crítica en un instante dado. Si dos procesos distintos estuvieran accediendo, al mismo tiempo, a una variable compartida para actualizarla, puede dar lugar a un abrazo mortal entre dichos procesos, y la ejecución del programa entraría en un ciclo interminable (bucle): la ejecución de un proceso espera la salida de otro proceso, y este a su vez espera una salida del anterior, en una reiteración infinita. En programación concurrente es esencial cuidar de no caer en un abrazo mortal. Y este es uno de los retos a salvar en la programación del SCC del futbol.

En [4] se introduce el SCC. Una jugada de pase se ejemplifica en la Fig. 1.

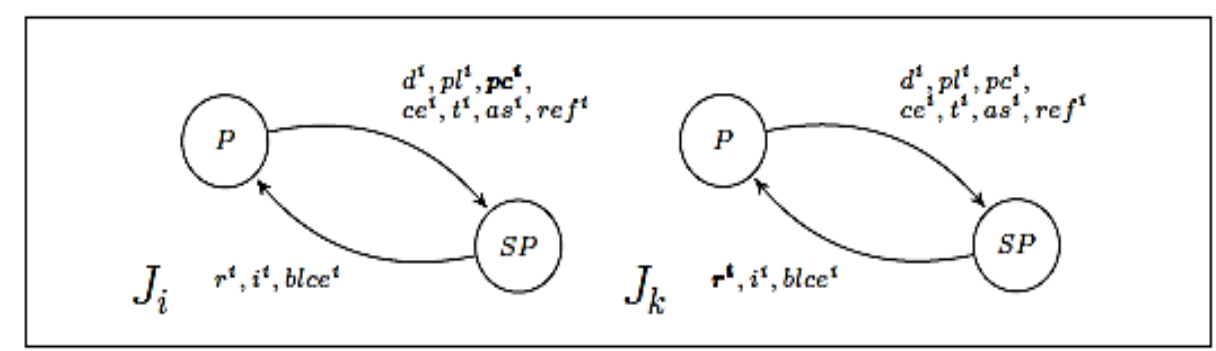

Fig. 1. Sistema concurrente de autómatas de dos jugadores para una jugada de pase corto [4]. 


\section{Selección de estrategias}

Para la elección de estrategias se asume la intención del entrenador y los jugadores de elegir las estrategias que dé lugar al mejor aporte de cada jugador en lo individual, pero, aún más, la que sea de mayor beneficio al equipo en lo colectivo, durante un juego. Así, la elección estratégica que proponemos utiliza el equilibrio de Nash (EN) [5]. Este método para elegir la estrategia tiene el efecto de minimizar el hacer jugadas poco exitosas o de riesgo para los jugadores en equipo durante un juego:

"Un perfil de estrategias es un equilibrio de Nash, si y solo si, para todo jugador y todas sus estrategias, la valoración del perfil actual es mayor o igual que el resto de los posibles perfiles"

Matemáticamente, es necesario definir una función de utilidad, por jugador, tal que evalué las estrategias del jugador con respecto al resto de los jugadores. Para formalizar el equilibrio de Nash introducimos la formulación de juego en forma normal.

\subsection{Juego en Forma Normal}

Sea $i \in\{1,2, \ldots, N\}$ un elemento en el conjunto de jugadores; sea la estrategia pura $\sigma_{k}^{i}$ la k-ésima acción del jugador $i$, tal que el conjunto de estrategias puras del jugador $i$ :

$$
D_{i}=\left\{\sigma_{1}^{i}, \sigma_{2}^{i}, \ldots, \sigma_{k}^{i}\right\}
$$

Sea $d$ un perfil de estrategia tal que:

$$
d \in D, D=D_{1} \times D_{2} \times \ldots \times D_{N} .
$$

Sea la función de utilidad $U_{i}: D \rightarrow \mathbb{R}$ que asigna un valor a cada perfil de estrategia conforme el jugador $i$. Normalizando el valor $U_{i}: D \rightarrow[0,1]$.

Un juego en Forma Normal se define como:

$$
G=\left\{D_{1}, \ldots, D_{N} ; U_{1}, \ldots, U_{N}\right\} .
$$

En un juego en forma normal se define una función de utilidad por jugador: cada jugador evalúa cada perfil de estrategias individualmente considerando su criterio. En el equilibrio de Nash, cada jugador, mediante su función de utilidad, evalúa los perfiles de estrategia y compara las evaluaciones entre sí.

\subsection{Equilibrio de Nash}

El equilibrio de Nash habilita un proceso de selección de perfiles de estrategia, siendo el criterio de selección de utilidad o máximo beneficio (menor riesgo) considerando las elecciones de los otros jugadores. Así, un perfil de estrategias $s^{*}=$ $\left(s_{1}^{*}, \ldots, s_{N}^{*}\right)$ satisface el EN sí y solo si: 


$$
\forall n, \forall s_{n} \neq s_{n}^{*}: U_{n}\left(s_{n}^{*}, s_{-n}^{*}\right) \geq U_{n}\left(s_{n}, s_{-n}^{*}\right)
$$

La función de utilidad $U_{i}: D \rightarrow \mathbb{R}$ para el futbol, valora los perfiles de estrategia con respecto a tres factores principales:

- Probabilidad de ocurrencia promedio por minuto (OPM) de jugadas.

- Distancias relativas entre jugadores.

- Habilidad o eficiencia de jugadores.

La OPM es analizada en [6]: es un promedio de las jugadas efectivas entre el total, y puede personalizarse a cada jugador. La habilidad de los jugadores se pondera considerando las estadísticas del mismo. Las jugadas son valoradas y ordenadas basadas en frecuencias de ocurrencias estadísticas abstraídas de juegos reales de beisbol. A partir de las estadísticas se definen las probabilidades de ocurrencia de las jugadas. Con base en la probabilidad se calcula la ocurrencia factual, efectiva, de cada jugada.

Cada rol de juego considera habilidades características para él, y tal que habilite para un mejor desempeño durante el juego. Los dos factores principales a considerar para la función de utilidad son la valoración $V_{i}\left(s_{k}^{i}\right)$ del jugador $i$ para la estrategia $s_{k}^{i}$, y la probabilidad $P_{i}\left(s_{k}^{i}\right)$ de su ocurrencia. La $P_{i}\left(s_{k}^{i}\right)$ de una estrategia se remite al estado actual del jugador. Esto es el proceso de elección aleatoria basado en la OPM. Cuando un jugador aplica su función de utilidad en un perfil de estrategias, está evaluado que tan benéfico es que el resto de los jugadores realicen la siguiente estrategia. Se propone una función de utilidad para cada rol de juego, similar a la definida en [2] para el futbol Americano. El valor obtenido a partir de un perfil de estrategias únicamente es comparable con los valores obtenidos por la misma función de utilidad.

\section{Discusión y trabajos relacionados}

En [7] el análisis estratégico del futbol es orientado a videojuegos utilizando algoritmos genéticos para generar oponentes que dependan de la habilidad del usuario y del nivel de juego. Un algoritmo genético utiliza técnicas inspiradas por la biología evolutiva tal como la selección natural, herencia, mutación y recombinación. La adecuación de cada estrategia del equipo es evaluada por la función de aptitud para enseñar como jugar de acuerdo a las reglas del futbol, y para evolucionar hacia mejores opciones estratégicas dadas por el valor de aptitud. En [3] la elección estratégica es iterando hacia atrás, desde el momento actual del partido $t_{i}, 1 \leq i$.

En [1] se utiliza el equilibrio de Nash para identificar los perfiles de estrategia preferidos por todos los jugadores del equipo tal que aumente la probabilidad de éxito: la cooperación enfatiza una participación positiva de los jugadores con base en la estrategia definida para obtener el triunfo de un partido. Cada perfil de estrategias es evaluado por la función de utilidad de cada jugador, para determinar los perfiles de estrategia que potencien el éxito del equipo. Así, cada perfil de estrategia es analizado y se consideran los que cumplen el equilibrio de Nash. El equilibrio de Nash toma las 
jugadas que sean más probables de ejecutarse con éxito, y así, mejora las opciones de éxito de un equipo.

Si bien el equilibrio de Nash toma las jugadas que sean más probables de ejecutarse con éxito, y así, mejora las opciones de éxito de un equipo. Computacionalmente, sin embargo, entre más jugadores participen en un juego y más estrategias puedan utilizar, la complejidad computacional de aplicar el equilibrio de Nash se incremente y pueda ser muy costoso. Más aun considerando la dinámica no pausada del futbol. Luego, aplicar el equilibrio de Nash, requiere combinarlo con heurísticas tal que disminuyan el dicho costo y sea factible utilizarlo para la toma de decisiones.

En [8], con el objetivo de reducir el tráfico en la red, [9] se enfoca en la aceleración del proceso de análisis por medio de la transferencia de equilibrio, o colocación de réplica (RPP). La transferencia de información sirve para determinar cuándo es conveniente replicar los datos dentro de un arreglo de servidores para acelerar el proceso que se atiende. A través de un análisis de costo de réplica y un proceso de minimización de costo de transferencia de objetos (OTC) se realiza la decisión estratégica para cada uno de los servidores. La función de utilidad determina el conjunto de estrategias factibles y se toma una decisión que minimice el costo de la réplica considerando a todos los agentes del sistema.

La idea clave en la transferencia de equilibrio es rehusar un equilibrio pre computado en juegos similares, ocurriendo después en el mismo estado si la pérdida de rehusar este estado puede ser tolerada. Para esto se deben tener en cuenta dos factores, la pérdida de la transferencia y la condición de transferencia. La pérdida de transferencia es la métrica de la desviación que puede surgir a partir de la reutilización de un equilibrio pre computado, la tolerabilidad de este factor debe ser configurada por el usuario, lo cual da a lugar a la condición de transferencia; si la perdida es menor al factor determinado por el usuario, entonces se puede realizar la transferencia.

En [9] el aprendizaje por refuerzo multiagente (MARL), es un proceso de decisión secuencial en problemas multiagente que en el entorno aprende a prueba y error. Los juegos de un solo tiro o no repetidos, como el futbol, en muchas ocasiones tienen un equilibrio similar en distintos momentos, lo cual permite reducir el número de cálculos por equilibrio, y así, la transferencia de equilibrio acelera el proceso.

Utilizando la transferencia de equilibrio, realizamos pruebas, simulando la posesión del balón y los pases entre jugadores. El campo de juego se representa en forma de una rejilla. Se limitan las acciones del juego a: arriba, abajo, izquierda, derecha y mantenerse quieto. De los experimentos realizados hasta ahora, pocas, pareciera eficiente aplicar la transferencia de equilibrio. Sin embargo, para sacar conclusiones se requieren muchas más pruebas.

\section{Conclusiones}

El lenguaje formal del futbol generado por una gramática libre de contexto habilita la descripción de cualquier combinación de jugadas y hasta de un partido completo. La interacción continúa es simulada por un sistema computacional concurrente. La habilidad futbolística de cada jugador y equipo se cuantifica en las estadísticas, las 
cuales, a su vez, dan lugar a la definición de una distribución de probabilidades para jugadores y equipos. El equilibrio de Nash como herramienta clásica de toma de decisiones, inclusive cooperativas, es aplicable para seleccionar estrategias de juego en el futbol. El número de jugadores, de jugadas posibles para cada uno y la cuasi absoluta continuidad del juego, lo hacen computacionalmente costoso de simular de manera exhaustiva. Es necesario complementar esta selección de estrategias con heurísticas y métodos que simplifiquen esta complejidad.

\section{Referencias}

1. Alvarado, M., Yee Rendon, A., Cocho, G.: Simulation of baseball gaming by cooperation and non-cooperation strategies. Computación y Sistemas, Vol. 18, No. 4, pp. 693-708 (2014)

2. Yee, A., Rodriguez, R., Alvarado, M.: Analysis of Strategies in American Football Using Nash Equilibrium. In: International Conference on Artificial Intelligence, Methodology, Systems, and Applications (2014)

3. Dobson, S., Goddard, J.: Optimizing strategic behaviour in a dynamic setting in professional team sports. European Journal of Operational Research, Vol. 205, No. 3, pp. 661-669 (2010)

4. Téllez, J., Alvarado, M.: Concurrency Simulation in Soccer. Social Robotics, Vol. 9979, No. 0302-9743, pp. 961-970 (2016)

5. Nash, J.: Non-cooperative games. Annals of mathematics, pp. 286-195 (1951)

6. Téllez, J., Alvarado, M.: Modelado y análisis formal de jugadas del fútbol. Research in Computing Science, Vol. 113, pp. 147-156 (2016)

7. Fernandez, A. J., Cotta, C., Ceballos, R. C.: Generating emergent team strategies in football simulation. GAMEON, pp. 120-128 (2008)

8. Khan, U. S., Ahmad, I.: A Pure Nash Equilibrium-Based Game Theoretical Method for Data Replication across Multiple Servers. IEEE Transactions on Knowledge and Data Engineering, Vol. 21, No. 4, pp. 537-553 (2009)

9. Hu, Y., Gao, Y., An, B.: Accelerating Multiagent Reinforcement Learning by Equilibrium Transfer. IEEE Transactions on Cybernetics, Vol. 45, No. 7, pp. 1289-1302 (2015) 